\title{
Dark matter mass from relic abundance, an extra $U(1)$ gauge boson, and active-sterile neutrino mixing
}

\author{
Imtiyaz Ahmad Bhat $\odot^{*}$ and Rathin Adhikari@ ${ }^{\dagger}$ \\ Centre for Theoretical Physics, Jamia Millia Islamia (Central University), \\ Jamia Nagar, New Delhi 110025, India
}

(Received 3 July 2019; revised manuscript received 30 January 2020; accepted 31 March 2020; published 15 April 2020)

\begin{abstract}
In a model with an extra $U(1)$ gauge to SM gauge group, we have shown the allowed region of masses of the extra gauge boson and the dark matter which is the lightest one among other right-handed Majorana fermions present in the model. To obtain this region, we have used bounds coming from constraints on activesterile neutrino masses and mixing from various oscillation experiments, constraint on dark matter relic density obtained by PLANCK together with the constraint on the extra gauge boson mass and its gauge coupling recently obtained by ATLAS Collaboration at LHC. From the allowed regions, it is possible to get some lower bounds on the masses of the extra gauge boson and the dark matter and considering those values it is possible to infer what could be the spontaneous symmetry breaking scale of an extra $U(1)$ gauge symmetry.
\end{abstract}

DOI: 10.1103/PhysRevD.101.075030

\section{INTRODUCTION}

Although Standard Model (SM) has got tremendous success in describing various phenomena at the elementary particle level, but SM failed to account for two major experimental results, one related to the existence of dark matter (DM) [1] in the Universe and the other related to neutrino oscillation phenomena that require neutrinos to be massive and significant mixings among different flavors of neutrinos. To accommodate neutrino masses and a viable dark matter candidate something beyond SM is necessary. One such example is minimal extension of SM gauge group with an extra $U(1)_{X}$ gauge symmetry. Additional symmetries [2] either global or gauged are imposed which play the role in guaranteeing the stability of dark matter candidate. There are several $U(1)_{X}$ gauge extended models with minimal extension to the SM [3-5]. An important feature of these models is that in comparison to SM there is one extra neutral gauge boson. In general, there could be mixing of the extra gauge boson $X$ with the SM $Z$ boson, which results in the modification of neutral current phenomena. The $Z$ pole data could be affected indirectly through such mixing and could shift the measured $Z$ mass and its coupling to SM fermions. But nice agreement of the mass and coupling with SM predictions constrains such mixing to be lower than $1 \%$ [6].

\footnotetext{
imtiyaz@ctp-jamia.res.in

rathin@ctp-jamia.res.in
}

Published by the American Physical Society under the terms of the Creative Commons Attribution 4.0 International license. Further distribution of this work must maintain attribution to the author(s) and the published article's title, journal citation, and DOI. Funded by SCOAP.
On the other hand, in cosmology to explain the rotational curves of the heavy massive body inside the galaxies, one need to propose the presence of dark matter $[7,8]$. Dark matter relic density has been constrained from PLANCK experiment [9].

$$
\Omega_{\mathrm{DM}} h^{2}=0.1200 \pm 0.0012
$$

where $\Omega_{\mathrm{DM}}$ is the density parameter for dark matter and $h=H_{0} /\left(100 \mathrm{~km} \mathrm{~s}^{-1} \mathrm{Mpc}^{-1}\right)$. Recently, CMS and ATLAS [10-12] Collaborations at LHC have obtained stringent bound on the mass and gauge coupling associated with the extra $U(1)$ gauge boson. In the light of recent neutrino oscillation phenomena $[13,14]$, there is a proposition of the presence of sterile neutrino apart from three active neutrinos. There are recent indications in the Fermilab experiment [15] about some nonzero mixing among active and sterile neutrinos with sterile neutrino mass in the $\mathrm{eV}$ scale [16].

In connection with these observational results, we have considered here an $U(1)$ gauge extended model [17], which contains dark matter fields and also can accommodate active-sterile neutrino masses and mixing. In this model, right-handed Majorana fermion is found to be a suitable candidate for dark matter as discussed later. There are some studies on the constraints on model parameters of $U(1)$ gauge extended models based on collider phenomenology and cosmological constraints [18]. However, in this work, we have shown in detail the allowed region in the dark matter mass $m_{\psi}$ and the extra $U(1)$ gauge boson mass $M_{X}$ plane. For that, we have considered PLANCK constraint on dark matter relic abundance. Besides, we consider constraints coming from active and sterile neutrino masses 
and their mixing to find the allowed region. In the model considered here, the presence of appropriate active and sterile neutrino mass and their mixing requires the presence of appropriate range of mixing (angle $\theta$ ) of dark matter with another right-handed Majorana fermion and their mass gap $\Delta$ as discussed later. In any extra $U(1)$ gauge model, the Majorana fermion (which is dark matter in our case) can be annihilated to SM fermion and antifermions through $X$ boson as mediator. However, in our work, due to activesterile mixing resulting in the nonzero value of $\theta$, the coannihilation of dark matter with other Majorana fermion is also present. So the observed dark matter relic density will depend on both annihilation and coannihilation of dark matter in general in our work. With LHC constraint along with relic abundance constraint and constraint on $\Delta$ and $\theta$ (from neutrino oscillation data), we have also studied the possibility of lower bounds on mass of $X$ boson and the dark matter mass and the corresponding $U(1)$ gauge coupling $g_{X}$.

Particularly, the allowed region is more for higher values of $M_{X}$ and $m_{\psi}$. It is found that the allowed regions do not significantly vary with $\Delta$ values but are more sensitive to the variations of $\theta$-the mixing of the dark matter with other heavy right-handed Majorana field considered in the model. All these analyses have been done considering $Z-X$ mixing to be zero at the tree level. Later on, we have shown that higher order corrections of this $Z-X$ mixing remain very small of the order of $10^{-5}$ and have been neglected.

In Sec. II, we have discussed the salient features of a model which is an $U(1)$ extension of the SM gauge group and the model can successfully explain active and sterile neutrino mass and mixing and also there is scope of dark matter. We have discussed the interaction of right-handed Majorana field in the mass basis with an extra gauge boson which will be useful for calculation of cross section for annihilation and coannihilation of dark matter. In Sec. III, using the experimental data on the active and sterile neutrino mass and their mixing, we have obtained the allowed region of the mass difference parameter $\Delta$ of dark matter with the next heavier right-handed Majorana fermion and their mixing angle $\theta$. In Sec. IV, dark matter relic density has been studied, taking into account annihilation as well as coannihilation of dark matter with next heavier right-handed Majorana fermion going into final states of SM fermion antifermion pair. In Sec. V, in finding allowed model parameters, we have considered certain allowed values of $\Delta$ and $\theta$ as obtained in Sec. III from active and sterile neutrino mass and mixing. We have obtained the allowed parameter space for dark matter mass $m_{\psi}, X$ boson mass $M_{X}$ based on constraints coming from LHC, and relic abundance and also neutrino oscillation mass and mixing constraint corresponding to various $\Delta$ and $\theta$ values. In Sec. VI, we have discussed possible modification of $Z-X$ mixing after including higher order correction. In Sec. VII, we have concluded our work.

\section{THE MODEL}

Here we have considered a model [17] which is an $U(1)$ extension of SM, in which neutrino masses have been studied extensively and the mass of neutrinos has been connected to dark matter which is stabilized by a residual $Z_{2}$ symmetry of the spontaneously broken $U(1)$ gauge symmetry. The model has only one electroweak symmetry breaking doublet $\phi^{(+, 0)}$ from which tree-level masses to quarks are obtained. Charged lepton masses are generated at one-loop level with dark matter as mediator. The three active and one sterile neutrino masses as well as mixing between active-sterile neutrinos have been generated through one loop. The model contains two heavy right-handed fermion triplet $\Sigma_{1 R, 2 R}^{(+, 0,-)}$ and three neutral singlet fermions $N_{R}, S_{1 R}$, $S_{2 R}$. These have been chosen so as to cancel all anomalies with each other. $U(1)_{X}$ gauge symmetry is spontaneously broken by singlet scalar $\chi_{1,2}^{0}$ and residual $Z_{2}$ symmetry is obtained. The other scalars are added to obtain masses for all fermions. There are two scalar doublets $\eta_{1,2}$-one couples to $S_{1 R}$ and other to two $\Sigma$ 's.

The fermionic and the scalar particles of the model are given in Tables I and II, respectively. Although there are several $U(1)$ charges corresponding to different fields but

TABLE I. Fermion fields in the model.

\begin{tabular}{lcc}
\hline \hline Particle & $U(1)_{X}$ & $Z_{2}$ \\
\hline$(u, d)_{L}$ & $n_{1}$ & + \\
$u_{R}$ & $\frac{1}{4}\left(7 n_{1}-3 n_{4}\right)$ & + \\
$d_{R}$ & $\frac{1}{4}\left(n_{1}+3 n_{4}\right)$ & + \\
$(\nu, l)_{L}$ & $n_{4}$ & + \\
$l_{R}$ & $\frac{1}{4}\left(-9 n_{1}+5 n_{4}\right)$ & + \\
$\Sigma_{1 R, 2 R}^{(+, 0,-)}$ & $\frac{3}{8}\left(3 n_{1}+n_{4}\right)$ & - \\
$N_{R}$ & $-\frac{3}{4}\left(3 n_{1}+n_{4}\right)$ & + \\
$S_{1 R}$ & $\frac{1}{8}\left(3 n_{1}+n_{4}\right)$ & - \\
$S_{2 R}$ & $\frac{5}{8}\left(3 n_{1}+n_{4}\right)$ & - \\
\hline \hline
\end{tabular}

TABLE II. Scalar fields in the model.

\begin{tabular}{lcr}
\hline \hline Particle & $U(1)_{X}$ & $Z_{2}$ \\
\hline$\phi^{(+, 0)}$ & $\frac{3}{4}\left(n_{1}-n_{4}\right)$ & + \\
$\eta_{1}^{(+, 0)}$ & $\frac{1}{8}\left(3 n_{1}-7 n_{4}\right)$ & - \\
$\eta_{2}^{(+, 0)}$ & $\frac{1}{8}\left(9 n_{1}-5 n_{4}\right)$ & - \\
$\chi_{1}^{0}$ & $\frac{1}{4}\left(3 n_{1}+n_{4}\right)$ & + \\
$\chi_{2}^{0}$ & $\frac{3}{4}\left(3 n_{1}+n_{4}\right)$ & + \\
$\chi_{3}^{0}$ & $\frac{1}{8}\left(3 n_{1}+n_{4}\right)$ & - \\
$\chi_{4}^{+}$ & $\frac{3}{8}\left(n_{1}-5 n_{4}\right)$ & - \\
$\xi^{(++,+, 0)}$ & $\frac{1}{8}\left(9 n_{1}-13 n_{4}\right)$ & - \\
\hline \hline
\end{tabular}


using the anomaly cancellation equations all of them can be expressed in terms of the other two $U(1)$ charges $n_{1}$ and $n_{4}$, corresponding to quark doublet and lepton doublet, respectively. Under $Z_{2}$ symmetry, odd and even fields are specified in the last column of the above tables. The relevant Yukawa Lagrangian part of the model is

$$
\begin{aligned}
L \supset & h_{i j}^{\Sigma} \Sigma_{i R} \Sigma_{j R} \overline{\chi_{2}^{0}}+h_{12}^{S} S_{1 R} S_{2 R} \overline{\chi_{2}^{0}}+h_{11}^{S} S_{1 R} S_{1 R} \overline{\chi_{1}^{0}} \\
& +h_{23}^{N} N_{R} S_{2 R} \chi_{3}^{0}+h_{i j}^{\eta_{i j}} \overline{\Sigma_{j R}^{0}} \nu_{i} \eta_{2}^{0}+h_{i 1}^{\eta_{1}} \overline{S_{1 R}} \nu_{i} \eta_{1}^{0} \\
& +h_{i j}^{\eta_{i}} l_{i L} \Sigma_{j R} \eta_{2}^{+}+h_{i j}^{\xi} l_{i R} \Sigma_{j R} \xi^{+}+h_{i 1}^{\eta_{1}} l_{i L} S_{1 R} \eta_{1}^{+} \\
& +h_{i 2}^{\chi} l_{i R} S_{2 R} \chi_{4}^{+} .
\end{aligned}
$$

The first six terms are relevant for masses of Majorana fermions (shown in Table 1), and also these terms are relevant for active-sterile mixing of neutrinos at one-loop level as discussed later. Last four Yukawa interactions are relevant for charged lepton mass generation and have not been discussed here. $\chi_{1,2}^{0}$, which breaks $U(1)_{X}$ gauge symmetry spontaneously, give masses to $\Sigma_{1 R, 2 R}^{(+, 0,-)}$ and $S_{1 R}, S_{2 R}$ through interaction as shown in first three terms above and so these scalars have even $Z_{2}$ parity. Also from these interactions, $U(1)_{X}$ charge of $\chi_{1,2}^{0}$ is specified by the corresponding $U(1)_{X}$ charge of triplet and singlet fermions as shown in the table. The active neutrinos get masses at one-loop level, through interactions shown in fifth and sixth terms in above interactions Lagrangian [17]. From $U(1)_{X}$ charges of the active neutrinos $\nu_{i}$ and the $S_{1 R}, S_{2 R}$ fermions, it follows that the scalar fields in fifth and sixth terms are different from $\chi_{1,2}^{0}$. They do not have nonzero vacuum expectation value (vev). So $\eta_{1,2}^{0}$ is considered an odd under $Z_{2}$. The SM fields are even under $Z_{2}$. So from fifth and sixth terms in above interactions, it follows that $\Sigma_{1 R, 2 R}^{(+, 0,-)}$ and $S_{1 R}, S_{2 R}$ are odd under $Z_{2}$. Interestingly, this oddness is decided by the $U(1)_{X}$ charge as discussed. Thus, these fermions could play the role of dark matter. From the required interactions for one-loop fermion masses for sterile neutrinos $N_{R}$ and charged leptons, the $Z_{2}$ parity of other nonstandard model fields is decided [17]. $N_{R}$ is the singlet neutrino which is massless at tree level. Mixing of $N_{R}$ with active neutrinos as shown later in Fig. 1 and also the mass of $N_{R}$ at one-loop level are obtained through interactions of $N_{R}$ with $S_{2 R}$ and a scalar field $\chi_{3}$. Here also scalar field different from $\chi_{1,2}$ is required because of the $U(1)_{X}$ charges of the fermions in this interaction and $\chi_{3}$ which does not have nonzero vev is required to be odd under $Z_{2}$. Then $N_{R}$ is required to be even under $Z_{2}$ and for that it is suitable for consideration as light sterile neutrino. The neutral scalars, odd under $Z_{2}$, in their mass basis have components, which are in general not electroweak singlets and as such they are not good dark matter candidate. This is because they will have too large cross section for their direct detection in underground experiments because of

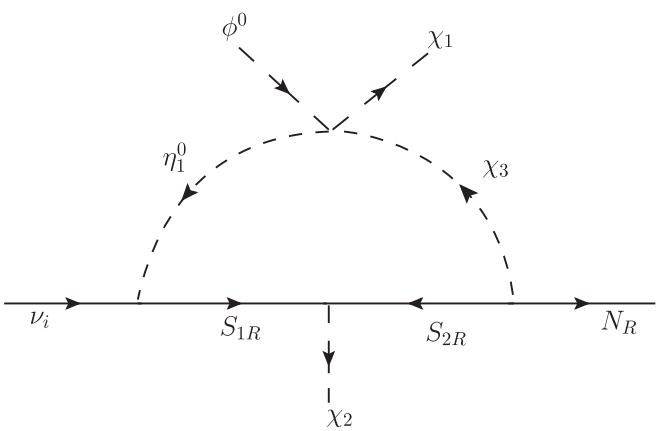

FIG. 1. One-loop active-sterile neutrino mixing [17].

their interactions with $Z$ boson. $\Sigma_{1 R, 2 R}^{0}$ as dark matter has been discussed in [19] and they do not play role in activesterile neutrino mixing. However, $S_{1 R, 2 R}$ could play the role of dark matter and also lead to active and sterile neutrino mixing as mediator at one loop and has been considered as possible dark matter candidate in our work.

We discuss in short the generation of mass of an extra gauge boson $X$ and its mixing with SM neutral gauge boson $Z$. Let the vevs of various neutral scalars fields be $\left\langle\phi^{0}\right\rangle=$ $v_{1}$ and $\left\langle\chi_{1,2}^{0}\right\rangle=u_{1,2}$, then the mass-squared elements, that determines mass for $Z$ and $X$, are given as

$M_{Z Z}^{2}=\frac{1}{2} g_{Z}^{2}\left(v_{1}^{2}\right)$,

$M_{Z X}^{2}=M_{X Z}^{2}=\frac{3}{8} g_{Z} g_{X}\left(n_{1}-n_{4}\right) v_{1}^{2}$,

$M_{X X}^{2}=\frac{1}{2} g_{X}^{2}\left(3 n_{1}+n_{4}\right)^{2}\left(u_{1}^{2}+9 u_{2}^{2}\right)+\frac{9}{8} g_{X}^{2}\left(n_{1}-n_{4}\right)^{2} v_{1}^{2}$.

Although in general, there is $Z-X$ mixing but it is expected to be very small so that electroweak precision measurements could be satisfied. The condition for no $Z-X$ mixing between neutral electroweak gauge boson and the extra $U(1)_{X}$ gauge boson is obtained for $M_{Z X}^{2}=0$ which gives $n_{1}=n_{4}$. With this zero mixing consideration, the mass of the extra $U(1)_{X}$ gauge boson is

$$
M_{X X}^{2}=\frac{1}{2} g_{X}^{2}\left(4 n_{1}\right)^{2}\left(u_{1}^{2}+9 u_{2}^{2}\right) .
$$

Later on, we consider this zero mixing condition in dark matter relic density calculation.

Since the dark matter is Majorana in nature, its vector coupling with $X$ boson is zero and it has only nonzero axialvector coupling with $X$. The vector coupling $g_{\mathrm{fv}}$ and axialvector coupling $g_{\mathrm{fa}}$ of the SM fermion fields with an extra gauge boson are given in Table III. These couplings are related to the chiral couplings [3] as follows:

$$
g_{f(v, a)}=\frac{1}{2}\left[\epsilon_{L}(f) \pm \epsilon_{R}(f)\right],
$$


TABLE III. Couplings of SM fermions with extra gauge boson $\mathrm{X}$ in terms of $U(1)$ charges $n_{1}$ and $n_{4} . U$ and $D$ are up and down type quarks, respectively.

\begin{tabular}{lcc}
\hline \hline & $g_{f v} / g_{X}$ & $g_{f a} / g_{X}$ \\
\hline$l=e, \mu, \tau$ & $\frac{9}{8}\left(n_{4}-n_{1}\right)$ & $\frac{1}{8}\left(n_{4}-9 n_{1}\right)$ \\
$\nu_{l}$ & $\frac{n_{4}}{2}$ & $-\frac{n_{4}}{2}$ \\
$U$ & $\frac{1}{8}\left(11 n_{1}-n_{4}\right)$ & $\frac{3}{8}\left(n_{1}-n_{4}\right)$ \\
$D$ & $\frac{1}{8}\left(5 n_{1}+3 n_{4}\right)$ & $\frac{3}{9}\left(n_{4}-n_{1}\right)$ \\
\hline \hline
\end{tabular}

where the chiral couplings $\epsilon_{L, R}(f)$ are $g_{X}$ times $U(1)_{X}$ charges corresponding to left- and right-handed chiral fields as shown in Table III.

Let the mass eigenstates of the four Majorana fermions $S_{1 R}, S_{2 R}, \Sigma_{1 R}^{0}, \Sigma_{2 R}^{0}$ be $\psi_{k}$ with mass $m_{\psi_{k}}$. The interaction basis $\psi_{j}^{\prime T}=\left[S_{1 R}, S_{2 R}, \Sigma_{1 R}^{0}, \Sigma_{2 R}^{0}\right]$ could be written in terms of this mass basis $\psi_{k}$ as

$$
\psi_{j}^{\prime}=\sum_{k} z_{j k} \psi_{k}
$$

with $j, k=1, \ldots 4$ where $\Sigma_{1 R}^{0}$ and $\Sigma_{2 R}^{0}$ are $S U(2)_{L}$ triplets and $S_{1 R}$ and $S_{2 R}$ are singlets. One of the lightest among $\psi_{k}$ say, $\psi_{1}$ is a dark matter candidate in this model, which we assume that it mainly contains $S_{1 R}$ and $S_{2 R}$. We consider $\psi_{2}$ as the next to lightest among these four mass eigenstates, and the masses $m_{\psi_{1}}$ and $m_{\psi_{2}}$ are not far apart.

In considering interactions of an extra gauge boson $\mathrm{X}$ with $S_{1 R}$ and $S_{2 R}$ in the mass basis of $\psi_{k}$, we are considering for simplicity that $z_{i j}$ mixing matrix elements have nonzero $1-2$ block with mixing angle $\theta$ which is decoupled from 3 to 4 block. Then the interaction can be written as

$$
\begin{aligned}
\sum_{i, j} & \overline{S_{i R}} \gamma_{\mu}\left(g_{i j} \gamma^{5}\right) S_{j R} X_{\mu} \\
= & \left(g_{S_{1 R} a} \cos ^{2} \theta+g_{S_{2 R} a} \sin ^{2} \theta\right) \overline{\psi_{1}} \gamma_{\mu} \gamma^{5} \psi_{1} X_{\mu} \\
& +\left(g_{S_{1 R} a} \sin ^{2} \theta+g_{S_{2 R} a} \cos ^{2} \theta\right) \overline{\psi_{2}} \gamma_{\mu} \gamma^{5} \psi_{2} X_{\mu} \\
& +\frac{1}{2} \sin 2 \theta\left(g_{S_{1 R} a}-g_{S_{2 R} a}\right) \overline{\psi_{1}} \gamma_{\mu} \gamma^{5} \psi_{2} X_{\mu} \\
& +\frac{1}{2} \sin 2 \theta\left(g_{S_{1 R} a}-g_{S_{2 R} a}\right) \overline{\psi_{2}} \gamma_{\mu} \gamma^{5} \psi_{1} X_{\mu},
\end{aligned}
$$

where $i, j=1,2, g_{S_{1 R} a}=5 / 8\left(3 n_{1}+n_{4}\right) g_{X}$, and $g_{S_{2 R} a}=$ $1 / 8\left(3 n_{1}+n_{4}\right) g_{X}$. Here $g_{X}$ is the gauge coupling for the extra gauge boson and the subscript $a$ denotes that these are axial-vector couplings. The interactions shown in terms of mass basis will be useful in Sec. IV in our calculation of cross section of annihilation and coannihilation of dark matter.

\section{ACTIVE AND STERILE NEUTRINO MASS AND MIXING}

There are eight real scalar fields, spanning $\sqrt{2} \operatorname{Re}\left(\eta_{1,2}^{0}\right)$, $\sqrt{2} \operatorname{Im}\left(\eta_{1,2}^{0}\right), \sqrt{2} \operatorname{Re}\left(\chi_{3}^{0}\right), \sqrt{2} \operatorname{Im}\left(\chi_{3}^{0}\right), \sqrt{2} \operatorname{Re}\left(\xi^{0}\right), \sqrt{2} \operatorname{Im}\left(\xi_{0}\right)$, with mass eigenstate as $\zeta_{l}$ with mass $m_{l}$. These fields are present in the one-loop diagram giving radiative masses to active and sterile neutrinos. For details about the one-loop diagrams giving masses to active and sterile neutrinos, we refer readers to Ref. [17]. However, we have shown the one-loop diagram in Fig. 1 gives rise to active and sterile neutrino mixing.

Apart from three light active neutrinos, $N_{R}$ plays the role of fourth neutrino as sterile in this model as mentioned earlier. The masses of active and sterile neutrinos are given as

$$
\begin{aligned}
\left(\mathcal{M}_{\nu}\right)_{i j}^{(2)}= & \frac{h_{i 1}^{\eta_{2}} h_{j 1}^{\eta_{2}}}{16 \pi^{2}} \sum_{k}\left(z_{3 k}\right)^{2} m_{\psi_{k}} A_{1} \\
& +\frac{h_{i 2}^{\eta_{2}} h_{j 2}^{\eta_{2}}}{16 \pi^{2}} \sum_{k}\left(z_{4 k}\right)^{2} m_{\psi_{k}} A_{2},
\end{aligned}
$$

where $A_{1}=\sum_{l}\left[\left(y_{2 l}^{R}\right)^{2} F\left(x_{l k}\right)-\left(y_{2 l}^{I}\right)^{2} F\left(x_{l k}\right)\right]$ and $A_{2}=$ $\sum_{l}\left[\left(y_{2 l}^{R}\right)^{2} F\left(x_{l k}\right)-\left(y_{2 l}^{I}\right)^{2} F\left(x_{l k}\right)\right] \quad \Sigma_{1 R}^{0}=\sum_{k} z_{3 k} \psi_{k}, \quad \Sigma_{2 R}^{0}=$ $\sum_{k} z_{4 k} \psi_{k}, \quad \sqrt{2} \operatorname{Re}\left(\eta_{2}^{0}\right)=\sum_{l} y_{2 l}^{R} \zeta_{l}, \quad \sqrt{2} \operatorname{Im}\left(\eta_{2}^{0}\right)=\sum_{l} y_{2 l}^{I} \zeta_{l}$, with $\sum_{k}\left(z_{3 k}\right)^{2}=\sum_{k}\left(z_{4 k}\right)^{2}=\sum_{l}\left(y_{2 l}^{R}\right)^{2}=\sum_{l}\left(y_{2 l}^{I}\right)^{2}=1$, and $x_{l k}=m_{l}^{2} / m_{\psi_{k}}^{2}$ and $F\left(x_{l k}\right)=x_{l k} \ln x_{l k} /\left(x_{l k}-1\right)$. Equation (9) is the contribution to the active neutrino masses from $\Sigma_{1 R}$ and $\Sigma_{2 R}$.

Let $\bar{S}_{1 R} \nu_{i} \eta_{1}^{0}$ coupling be $h_{i 1}^{\eta_{1}}$, then the contribution to $\mathcal{M}_{\nu}$ is given by

$$
\left(\mathcal{M}_{\nu}\right)_{i j}=\frac{h_{i 1}^{\eta_{1}} h_{j 1}^{\eta_{1}}}{16 \pi^{2}} \sum_{k}\left(z_{1 k}\right)^{2} m_{\psi_{k}} A,
$$

where $A=\sum_{l}\left[\left(y_{1 l}^{R}\right)^{2} F\left(x_{l k}\right)-\left(y_{1 l}^{I}\right)^{2} F\left(x_{l k}\right)\right]$, and $S_{1 R}=$ $\sum_{k} z_{1 k} \psi_{k}, \quad \sqrt{2} \operatorname{Re}\left(\eta_{1}^{0}\right)=\sum_{l} y_{1 l}^{R} \zeta_{l}, \quad \sqrt{2} \operatorname{Im}\left(\eta_{1}^{0}\right)=\sum_{l} y_{1 l}^{I} \zeta_{l}$, with $\sum_{k}\left(z_{1 k}\right)^{2}=\sum_{l}\left(y_{1 l}^{R}\right)^{2}=\sum_{l}\left(y_{1 l}^{I}\right)^{2}=1$. We have assumed that the first contribution to active neutrino mass shown in Eq. (9) is somewhat lesser than the second contribution shown in Eq. (10) which gives masses to heavier neutrinos and the combination gives rise to appropriate mixing among different active neutrinos. We have considered only the mass scale for active neutrinos in our work and will be concerned with only Eq. (10).

Let $S_{2 R} N_{R} \chi_{3}^{0}$ coupling be $h_{23}^{N}$, then the mass of sterile neutrino is given as

$$
m_{N N}=\frac{h_{23}^{N} h_{23}^{N}}{16 \pi^{2}} \sum_{k}\left(z_{2 k}\right)^{2} m_{\psi_{k}} B
$$

where $B=\sum_{l}\left[\left(y_{3 l}^{R}\right)^{2} F\left(x_{l k}\right)-\left(y_{3 l}^{I}\right)^{2} F\left(x_{l k}\right)\right]$ and $S_{2 R}=$ $\sum_{k} z_{2 k} \psi_{k}, \sqrt{2} \operatorname{Re}\left(\chi_{3}^{0}\right)=\sum_{l} y_{3 l}^{R} \zeta_{l}, \quad \sqrt{2} \operatorname{Im}\left(\chi_{3}^{0}\right)=\sum_{l} y_{3 l}^{I} \zeta_{l}$, with $\sum_{k}\left(z_{2 k}\right)^{2}=\sum_{l}\left(y_{3 l}^{R}\right)^{2}=\sum_{l}\left(y_{3 l}^{I}\right)^{2}=1$. 
The active-sterile neutrino mixing is possible because of interaction $h_{12}^{S} S_{1 R} S_{2 R} \chi_{2}^{0}$ as shown in Fig. 1, and nondiagonal mass matrix elements related to mixing in active-sterile neutrino mass matrix are given as

$$
m_{\nu N}=\frac{h_{i 1}^{\eta_{1}}\left(h_{23}^{N}\right)}{16 \pi^{2}} \sum_{k} z_{1 k} z_{2 k} m_{\psi_{k}} C
$$

where $C=\sum_{l}\left[y_{1 L}^{R} y_{3 l}^{R} F\left(x_{l k}\right)-y_{1 l}^{I} y_{3 l}^{I} F\left(x_{l k}\right]\right.$ and $\sum_{k} z_{1 k} z_{2 k}=$ $\sum_{l} y_{1 l}^{R} y_{3 l}^{R}=\sum_{l} y_{1 l}^{I} y_{3 l}^{I}=0$. A, B, and $C$ are loop factors corresponding to the one-loop diagrams that give masses and mixing of neutrinos. Based on the recent global fit [20] of neutrino oscillation experiment with sterile neutrino in $3+1$ scheme, the best fit values are $\Delta m_{41}^{2}=1.3 \mathrm{eV}^{2}$, $\left|U_{e 4}\right|=0.1$, and $\left|U_{\mu 4}\right| \lesssim 10^{-2}$. Also, taking into account the cosmological constraint on sum of three active neutrino masses [21,22], we consider active neutrino masses, sterile neutrino mass, and active-sterile mixing as

$$
\begin{aligned}
\left(\mathcal{M}_{\nu}\right)_{i j} & \sim 0.1 \mathrm{eV}, \quad \mathcal{M}_{N N} \sim 1.14 \mathrm{eV} \\
\mathcal{M}_{\nu N} & \sim 0.114 \mathrm{eV} .
\end{aligned}
$$

The product of the mixing matrix element and $m_{\psi k}$ which are present in Eqs. (10)-(12) can be rewritten in terms of the mixing angle $\theta$ and the mass gap parameter $\Delta=\left(m_{\psi_{2}}-m_{\psi_{1}}\right) / m_{\psi_{1}}$ after we consider $z_{11}=\cos \theta$, $z_{12}=-\sin \theta, \quad z_{21}=\sin \theta$, and $z_{22}=\cos \theta$. Following these, we can write

$$
\begin{aligned}
\sum_{k} z_{1 k} z_{2 k} m_{\psi_{k}} & =\frac{\Delta m_{\psi_{1}} \sin 2 \theta}{2} \\
\sum_{k}\left(z_{2 k}\right)^{2} m_{\psi_{k}} & =m_{\psi_{1}}\left(1+\Delta \cos ^{2} \theta\right) \\
\sum_{k}\left(z_{1 k}\right)^{2} m_{\psi_{k}} & =m_{\psi_{1}}\left(1+\Delta \sin ^{2} \theta\right) .
\end{aligned}
$$

Using Eqs. (13) and (14) for active neutrino mass scale and the mixing of sterile neutrino $U_{e 4}$, we can write Eqs. (10)(12) in terms of $\Delta$ and $\theta$ parameters and can be written as

$$
\begin{aligned}
\frac{h_{11}^{\eta_{1}} h_{11}^{\eta_{1}}}{16 \pi^{2}} m_{\psi_{1}}\left(1+\Delta \sin ^{2} \theta\right) & A \approx 0.1, \\
\frac{h_{23}^{N} h_{23}^{N}}{16 \pi^{2}} m_{\psi_{1}}\left(1+\Delta \cos ^{2} \theta\right) & B \approx 1.14, \\
\left.\frac{h_{11}^{\eta_{1}} h_{23}^{N}}{16 \pi^{2}} m_{\psi_{1}} \frac{(\Delta \sin 2 \theta}{2}\right) & C \approx 0.1 .
\end{aligned}
$$

If we consider $\sum z^{2} m_{\psi_{k}}$ of Eq. (14) of the order of $1 \mathrm{TeV}$ and couplings $h \sim 0.1$, then from Eqs. (15)-(17), it is found that $A, B$, and $C$ are in the range of $10^{-8}$ to $10^{-9}$. However, considering the variation of two couplings $h_{11}^{\eta_{1}}$ and $h_{23}^{N}$ in

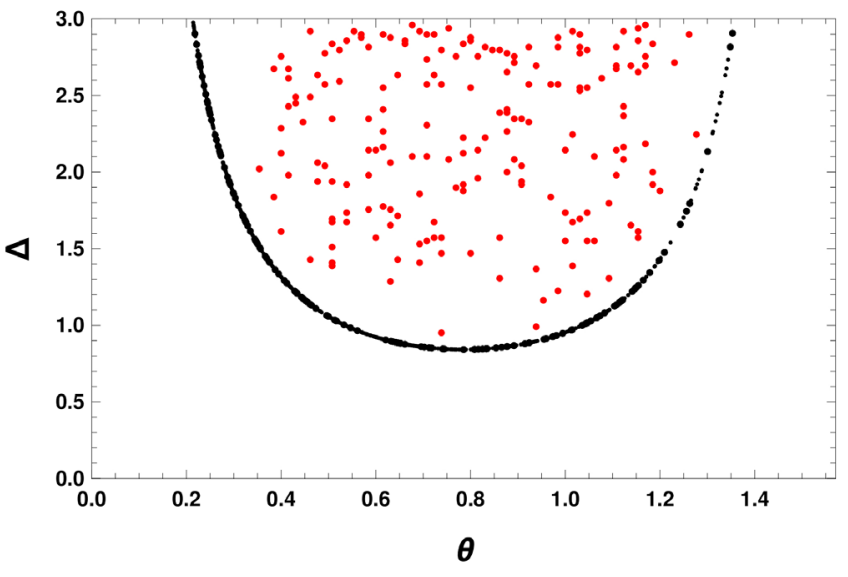

FIG. 2. Allowed region of $\Delta$ and $\theta$ (in radian) from activesterile neutrino masses and mixing.

the range of $0.05-1$ and $A, B$, and $C$ in the range of $10^{-9}-$ $10^{-6}$ in Eqs. (15)-(17), we obtain the allowed region of $\theta$ and $\Delta$ as shown in Fig. 2. In Fig. 2, we consider two different conditions among $A, B$, and $C$ : (1) $A=B=C$ and (2) $C<A, B$. The condition (1) gives the allowed almost semicircle outer line, whereas condition (2) gives region inside covered by that almost semicircle line. One may note here that $A$ and $B$ as mentioned just after Eqs. (10) and (11) are very similar in nature with the sum over the product of the mixing matrix elements of $y$ being 1 for both $\mathrm{A}$ and $\mathrm{B}$, whereas for $C$ as mentioned just after Eq. (12) due to orthogonality condition the sum over the product of the mixing matrix elements of $y$ vanishes. Due to this difference, $C$ is expected to be lesser than both $A$ and $B$. In that sense, we should consider the proper allowed region of $\Delta$ and $\theta$ as that given by the region inside the almost semicircle line in Fig. 2 and $\Delta \geq 1$ is found to be preferred.

For active and sterile neutrino mixing, one important conclusion follows from both Figs. 1 and 2 is that there is necessarily nonzero mixing $\theta$ between $S_{1 R}$ and $S_{2 R}$; otherwise, there will be zero contribution from Fig. 1 . Figure 2, however, shows apart from nonzero mixing, the simultaneous constraint on both $\theta$ and $\Delta$. These imply that apart from annihilation of two dark matter fields $\left(\psi_{1}\right)$ into SM fermion and antifermion pair, there is coannihilation of dark matter field $\psi_{1}$ with other next heavier Majorana field $\psi_{2}$ through the interactions mentioned in Eq. (8).

\section{DARK MATTER $\left(\psi_{1}\right)$ RELIC DENSITY}

Relic density is obtained from the Boltzmann equation [23] governing the evolution of number density of the DM with the thermally averaged cross section for the process $\psi_{1} \psi_{1} \rightarrow f \bar{f}$. The Boltzmann equation is written as

$$
n_{\psi_{1}}^{\cdot}+3 H n_{\psi_{1}}=\langle\sigma v\rangle\left(\left(n_{\psi_{1}}^{\mathrm{eqb}}\right)^{2}-n_{\psi_{1}}^{2}\right),
$$


where $n_{\psi_{1}}$ is the number density and $n_{\psi_{1}}^{\text {eqb }}$ is thermal equilibrium number density of the DM particle. $H$ is Hubble expansion rate of the Universe, and $\langle\sigma v\rangle$ is the thermally averaged cross section for the process $\psi_{1} \psi_{1} \rightarrow$ $f \bar{f}$ and is given by [24]

$\langle\sigma v\rangle=\frac{1}{8 m_{\psi_{1}}^{4} T K_{2}^{2}(m / T)} \int_{4 m_{\psi_{1}}^{2}}^{\infty} \sigma\left(s-4 m_{\psi_{1}}^{2}\right) \sqrt{s} K_{1}(\sqrt{s} / T) d s$,

where $K_{1}, K_{2}$ are modified Bessel functions of first and second kinds, respectively. Here $s$ is the center of mass energy squared. The thermally averaged cross section can be expanded in powers of relative velocity of two dark matter particle to be scattered and is written as $\langle\sigma v\rangle=$ $a+b v^{2}$. Numerical solution of the above Boltzmann equation gives [25]

$$
\Omega_{\psi_{1}} h^{2} \approx \frac{1.04 \times 10^{9} x_{f}}{M_{\mathrm{pl}} \sqrt{g_{*}}\left(a+3 b / x_{f}\right)},
$$

where $x_{f}=m_{\psi_{1}} / T_{f}, T_{f}$ is the freeze-out temperature, $g_{*}$ is the number of relativistic degrees of freedom at the time of freeze-out. $x_{f}$ can be found from

$$
x_{f}=\ln \frac{0.038 M_{\mathrm{Pl}} m_{\psi_{1}}\langle\sigma v\rangle}{g_{*}^{1 / 2} x_{f}^{1 / 2}} .
$$

We assume $\psi_{1}$ to be lighter than $\psi_{2}$ and it plays the role of dark matter. The lighter $\psi_{1}$ will be pair annihilated to SM fermions and antifermions through the extra gauge boson mediator but not $Z$ boson as we are considering $Z-X$ mixing to be zero. This pair annihilation has been considered above. However, it could also coannihilate with $\psi_{2}$. Both annihilation and coannihilation cross sections could control the relic abundance of the dark matter $\psi_{1}[26,27]$. To take into account coannihilation, we discuss the necessary modifications in the Boltzmann equation below.

If the mass difference between $\psi_{1}$ and $\psi_{2}$ is very large, $\psi_{2}$ will be out of thermal equilibrium much earlier than $\psi_{1}$ and coannihilation will not play a significant role in the evolution of the number density of $\psi_{1}$. However, we consider the case where the mass difference may not to be too large. In that case, we consider the annihilation as well as the coannihilation channel in the coupled Boltzmann equation to find out the evolution of the number density of $\psi_{1}$ and hence find the relic density of dark matter. Using the formalism of Ref. [26], we can reduce the system of two Boltzmann equations governing number densities $n_{1}$ and $n_{2}$ of $\psi_{1}$ and $\psi_{2}$, respectively, into one Boltzmann equation which governs the evolution of $n=n_{1}+n_{2}$ in the early Universe as given below.

$$
\dot{n}=-3 H n-\sum_{i, j=1}^{2}\left\langle\sigma_{i j} v\right\rangle\left(\left(n_{i} n_{j}-n_{i}^{\mathrm{eq}} n_{j}^{\mathrm{eq}}\right),\right.
$$

where $\left\langle\sigma_{i j} v\right\rangle$ is the thermally averaged scattering cross section for the process $\psi_{i} \psi_{j} \rightarrow f \bar{f}$. This equation can be further simplified as

$$
\dot{n}=-3 H n-\sum_{i, j=1}^{2}\left\langle\sigma_{\mathrm{eff}} v\right\rangle\left(\left(n^{2}-\left(n^{\mathrm{eq}}\right)^{2}\right),\right.
$$

where $\sigma_{\text {eff }}$ is given as

$\sigma_{\mathrm{eff}} \approx \sum_{i, j=1}^{2} \sigma_{i j} \frac{g_{i} g_{j}}{g_{\mathrm{eff}}^{2}}\left(1+\Delta_{i}\right)^{3 / 2}\left(1+\Delta_{j}\right)^{3 / 2} \exp ^{-x\left(\Delta_{i}+\Delta_{j}\right)}$.

Here $x=m_{\psi_{1}} / T$ and $\Delta_{i}=\frac{m_{\psi_{i}}-m_{\psi_{1}}}{m_{\psi_{1}}}$. Then $\Delta_{1}=0$ by definition. Later on, $\Delta_{2}$ is written as $\Delta . g_{i}$ is the internal degrees of freedom of the interacting particles and $g_{\text {eff }}$ is defined as

$$
g_{\mathrm{eff}}=\sum_{i=1}^{2} g_{i}\left(1+\Delta_{i}\right) 3 / 2 \exp ^{-x \Delta_{i}}
$$

For comparison with the general weakly interacting massive particle formulas, we have Taylor expanded the thermally averaged cross sections,

$\left\langle\sigma_{i j} v\right\rangle=a_{i j}+b_{i j} v^{2} ; \quad\left\langle\sigma_{\mathrm{eff}} v\right\rangle=a_{\mathrm{eff}}+b_{\mathrm{eff}} v^{2}$,

where $a_{\text {eff }}$ and $b_{\text {eff }}$ are given by

$a_{\mathrm{eff}} \approx \sum_{i, j=1}^{2} a_{i j} \frac{g_{i} g_{j}}{g_{\mathrm{eff}}^{2}}\left(1+\Delta_{i}\right)^{3 / 2}\left(1+\Delta_{j}\right)^{3 / 2} \exp ^{-x\left(\Delta_{i}+\Delta_{j}\right)}$,
$b_{\mathrm{eff}} \approx \sum_{i, j=1}^{2} b_{i j} \frac{g_{i} g_{j}}{g_{\mathrm{eff}}^{2}}\left(1+\Delta_{i}\right)^{3 / 2}\left(1+\Delta_{j}\right)^{3 / 2} \exp ^{-x\left(\Delta_{i}+\Delta_{j}\right)}$.

The phase space integration part for all the process of $\psi_{i} \psi_{j} \rightarrow f \bar{f}$ is almost same for cross section calculation, and the difference in the cross sections is mainly due to the strength of couplings $g_{i j}$ in which both the indices $i, j$ run from 1 to 2. Because of this, we can write

$$
\frac{\sigma_{11}}{\left|g_{11}\right|^{2}} \approx \frac{\sigma_{12}}{\left|g_{12}\right|^{2}} \approx \frac{\sigma_{21}}{\left|g_{21}\right|^{2}} \approx \frac{\sigma_{22}}{\left|g_{22}\right|^{2}}
$$

Using this approximation $\sigma_{\text {eff }}$ in Eq. (24), it can be written in terms of $\sigma_{11}$ as

$$
\sigma_{\text {eff }}=\frac{g_{i} g_{j}}{g_{\text {eff }}^{2}}\left[1+2 r_{12} \frac{g_{12}^{2}}{g_{11}^{2}}+r_{22} \frac{g_{22}^{2}}{g_{11}^{2}}\right] \sigma_{11} .
$$


Here, $\quad r_{12}=(1+\Delta)^{3 / 2} e^{-x \Delta}, \quad r_{22}=(1+\Delta)^{3} e^{-2 x \Delta}$, and $\Delta=\left(m_{\psi_{2}}-m_{\psi_{1}}\right) / m_{\psi_{1}}$ and $\sigma_{11}$ is annihilation cross section of $\psi_{1} \psi_{1} \rightarrow f \bar{f}$, and $\left\langle\sigma_{11} v\right\rangle$ can be Taylor expanded in the form of $a_{11}+b_{11} v^{2}$. For Majorana fermions $g_{i}=g_{j}=2$ (internal degrees of freedom) and from Eq. (8), the couplings involved in these annihilation and coannihilation channels are

$$
\begin{aligned}
& g_{11}=g_{S_{1 R} a} \cos ^{2} \theta+g_{S_{2 R} a} \sin ^{2} \theta \\
& g_{22}=g_{S_{2 R} a} \cos ^{2} \theta+g_{S_{1 R} a} \sin ^{2} \theta \\
& g_{12}=g_{21}=\frac{1}{2} \sin 2 \theta\left(g_{S_{1 R} a}-g_{S_{2 R} a}\right) .
\end{aligned}
$$

In the presence of coannihilation of dark matter $\psi_{1}$ with $\psi_{2}$, Eqs. (20) and (21) will be modified as

$$
\Omega_{\psi_{1}} h^{2} \approx \frac{1.04 \times 10^{9} x_{f}}{M_{\mathrm{pl}} \sqrt{g_{*}}\left(a_{11} I_{a}+3 b_{11} I_{b} / x_{f}\right)},
$$

where

$I_{a}=\frac{x_{f}}{a_{11}} \int_{x_{f}}^{\infty} x^{-2} a_{\mathrm{eff}} d x$ and $I_{b}=\frac{2 x_{f}^{2}}{b_{11}} \int_{x_{f}}^{\infty} x^{-3} b_{\mathrm{eff}} d x$

and $x_{f}$ can be obtained from

$$
x_{f}=\ln \frac{0.038 M_{\mathrm{Pl}} m_{\psi_{1}}\left\langle\sigma_{\mathrm{eff}} v\right\rangle}{g_{*}^{1 / 2} x_{f}^{1 / 2}} .
$$

Following [28], the annihilation cross section of Majorana fermion to SM $f \bar{f}$ through s channel mediated by $\mathrm{X}$ boson is given as

$$
\begin{aligned}
\sigma_{11}= & \frac{n_{c}}{12 \pi s\left[\left(s-M_{X}^{2}\right)^{2}+M_{X}^{2} \Gamma_{X}^{2}\right]} \sum_{f} \sqrt{\frac{1-4 m_{f}^{2} / s}{1-4 m_{\psi_{1}}^{2} / s}} \\
& \times\left[g _ { f a } ^ { 2 } g _ { 1 1 } ^ { 2 } \left(4 m_{\psi_{1}}^{2}\left[m_{f}^{2}\left(7-\frac{6 s}{M_{X}^{2}}+\frac{3 s^{2}}{M_{X}^{4}}\right)-s\right]\right.\right. \\
& \left.\left.+s\left(s-4 m_{f}^{2}\right)\right)+g_{f v}^{2} g_{11}^{2}\left(s+2 m_{f}^{2}\right)\left(s-4 m_{\psi_{1}}^{2}\right)\right],
\end{aligned}
$$

where $n_{c}=3$ when $f$ stands for quarks and $n_{c}=1$ when $f$ stands for leptons and $s \approx 4 m_{\psi_{1}}^{2}+m_{\psi_{1}}^{2} v^{2}$. In the above equation, $\Gamma_{X}=\Gamma_{X_{1}}+\Gamma_{X_{2}}$ is the total decay width of an extra gauge boson $X$, where $\Gamma_{X_{1}}$ is the decay width of the extra gauge boson $X$ to SM fermion antifermion pair and $\Gamma_{X_{2}}$ is the decay width of the extra gauge boson decaying to $\psi_{i} \psi_{j}$ Majorana fermions,

$$
\begin{aligned}
\Gamma_{X_{1}} & \equiv \sum_{f} \Gamma(X \rightarrow f \bar{f}) \\
& =\sum_{f} \frac{n_{c} M_{X}}{12 \pi S} \sqrt{1-\frac{4 m_{f}^{2}}{M_{X}^{2}}}\left[g_{\mathrm{fa}}^{2}\left(1-\frac{4 m_{f}^{2}}{M_{X}^{2}}\right)+g_{\mathrm{fv}}^{2}\left(1-2 \frac{m_{f}^{2}}{M_{X}^{2}}\right)\right] \\
\Gamma_{X_{2}} & =\sum_{i, j} \Gamma\left(X \rightarrow \psi_{i} \bar{\psi}_{j}\right)=\sum_{i, j} \frac{M_{X} g_{i j}^{2}}{12 \pi S}\left[1-\frac{4 m_{\psi_{i}} m_{\psi_{j}}}{M_{X}^{2}}\right]^{3 / 2},
\end{aligned}
$$

where $S=1$ (2) for (in)distinguishable final state particles. In the notation of Eq. (26), $\left\langle\sigma_{11} v\right\rangle=a_{11}+b_{11} v^{2}$, where

$$
\begin{aligned}
a_{11}= & \frac{n_{c} g_{f a}^{2} m_{f}^{2} g_{11}^{2} m_{\psi_{1}}}{24 \pi\left[\left(M_{X}^{2}-4 m_{\psi_{1}}^{2}\right)^{2}+M_{X}^{2} \Gamma_{X}^{2}\right]} \\
& \times \sqrt{1-\frac{m_{f}^{2}}{m_{\psi_{1}}^{2}}\left(12-96 \frac{m_{\psi_{1}}^{2}}{M_{X}^{2}}+192 \frac{m_{\psi_{1}}^{4}}{M_{X}^{4}}\right),} \\
b_{11}= & a_{11}\left[-\frac{1}{4}+\frac{m_{f}^{2}}{8\left(m_{\psi_{1}}^{2}-m_{f}^{2}\right)}-\frac{M_{X}^{4}-16 M_{X}^{2} m_{\psi_{1}}^{2}+48 m_{\psi_{1}}^{4}}{4\left(\left(M_{X}^{2}-4 m_{\psi_{1}}^{2}\right)^{2}+M_{X}^{2} \Gamma_{X}^{2}\right)}\right. \\
& \left.+\frac{\left(-4+2 \frac{g_{f v}^{2}}{g_{f a}^{2}}+4 \frac{m_{\psi_{1}}^{2}}{m_{f}^{2}}+4 \frac{g_{f v}^{2} m_{\psi_{1}}^{2}}{g_{f a}^{2} m_{f}^{2}}-24 \frac{m_{\psi_{1}}^{2}}{M_{X}^{2}}+96 \frac{m_{\psi_{1}}^{4}}{M_{X}^{4}}\right)}{\left(12-96 \frac{m_{\psi_{1}}^{2}}{M_{X}^{2}}+192 \frac{m_{\psi_{1}}^{4}}{M_{X}^{4}}\right)}\right] .
\end{aligned}
$$

Mass $M_{X}$ of the extra gauge boson in the above expressions is given by Eq. (5), and $g_{11}$ corresponds to axial-vector coupling as follows from Eqs. (31) and (8).

\section{ALLOWED REGION OF DARK MATTER MASS, $X$ BOSON MASS, AND ITS GAUGE COUPLING}

Large Hadron Collider (LHC) operating at $\sqrt{s}=$ $13 \mathrm{TeV}$ has searched for new phenomena [10-12] which are resonant as well as nonresonant and in which the final state is dilepton/dijet. This is a robust test for all theories beyond the SM. ATLAS at LHC has obtained the allowed region of coupling $g_{X}$ of quarks and leptons with an extra gauge boson mass $M_{X}$ in Fig. 4 of [10] and Fig. 5(a) of [11] at $95 \%$ confidence level. However, these two figures in Refs. $[10,11]$ do not differ too much. We have considered the allowed region of $g_{X}$ and $M_{X}$ in Fig. 5(a) of Ref. [11] in our numerical analysis. $g_{X}$ coupling in our paper is related to coupling $\gamma^{\prime}$ of this figure as $g_{X} \approx 0.463 \gamma^{\prime}$.

For our numerical analysis, $U(1)$ charges are fixed by considering $n_{1}=n_{4}=1 / \sqrt{2}$ (normalizing $n_{1}^{2}+n_{4}^{2}=1$ ) which satisfies zero mixing condition of an extra gauge boson $X$ with SM $Z$ boson. We have considered two values of $\Delta$ as 1 as 1 and 2 and also different values of $\theta$ as 0.4 , $\pi / 4$, and 1.2 as allowed by Fig. 2 . 


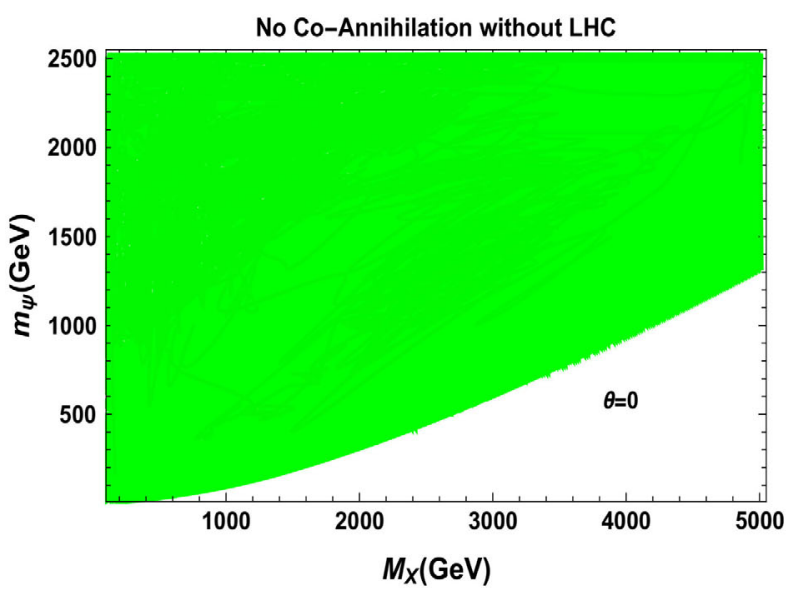

(a)

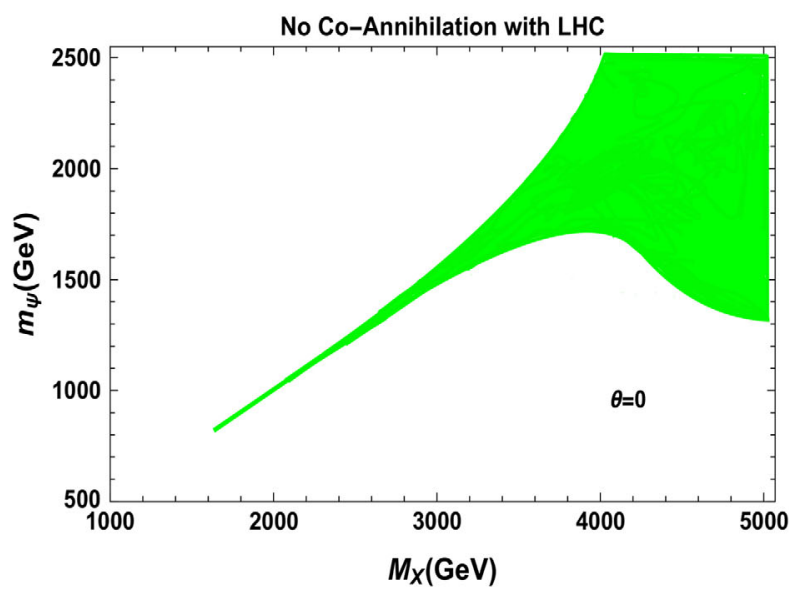

(b)

FIG. 3. Allowed green region in the $M_{X}$ and $m_{\psi}$ plane for the no coannihilation channel satisfies PLANCK relic density bound $\Omega h^{2} \in(0.1188,0.1212)$. (a),(b) correspond to no LHC constraints and LHC constraints on $M_{X}$ and $g_{X}$, respectively.

In the later part, we have used the symbol $m_{\psi}$ for the dark matter mass $m_{\psi_{1}}$. In Fig. 3, we first show the allowed region in $M_{X}-m_{\psi}$ plane for only the annihilation channel $\left(\psi_{1} \psi_{1} \rightarrow f \bar{f}\right)$ but no coannihilation of dark matter fermion. As discussed earlier, this will not in general correspond to active and sterile neutrino mixing in the model considered by us because of the no coannihilation channel. For comparing the allowed region with and without LHC constraint, as shown in Fig. 3, we have considered the variation of $g_{X}$ over the range $(0.005-0.7), m_{\psi}$ up to $2.5 \mathrm{TeV}$, and $M_{X}$ up to $5 \mathrm{TeV}$, as this is the range considered by LHC. The other two parameters $\theta$ and $\Delta$ are zero as this figure is only for the annihilation case. In plotting Fig. 3(a), we have considered only the constraint coming from relic abundance on dark matter from PLANCK $2018 \Omega h^{2} \in$ $(0.1188,0.1212)$ [9]. But, in Fig. 3(b), we have also considered the constraint on $g_{X}$ and $M_{X}$ given by ATLAS Collaboration [10,11] at LHC. Comparing Figs. 3(a) and 3(b), one can see that LHC constraint significantly reduces the allowed region of dark matter mass for lower values of $M_{X}<4000 \mathrm{GeV}$ and for further lower $M_{X}$ the allowed range of dark matter mass $m_{\psi}$ is further constrained with respect to no LHC constraint in Fig. 3(a). For $M_{X}$ lesser than about $1600 \mathrm{GeV}$ and $m_{\psi}$ lesser than about $700 \mathrm{GeV}$, it is difficult to get any allowed region.

In Fig. 4, apart from considering the constraint coming from relic abundance on dark matter from Planck 2018, we have also considered the coannihilation channel along with the annihilation channel for the dark matter as required for satisfying active and sterile neutrino masses and mixing. As discussed in Sec. III, the preferred allowed region of $\Delta$ and $\theta$ is shown in Fig. 2 inside the almost semicircular line. We consider in Fig. 4 different values of $\Delta$ and $\theta$ based on Fig. 2. But no LHC constraint has been imposed.

One can see after including the coannihilation channel for all cases in Fig. 4 there is a significant constraint on allowed dark matter mass for any $M_{X}$ value in comparison to Fig. 3(a) with no coannihilation for which $\theta=0$. A significant change has come mainly due to nonzero $\theta$ value considered in Fig. 4 as the coupling $g_{i j}$ of $\psi_{i} \psi_{j} X$ as shown in Eqs. (8) and (31) changes with the change in $\theta$ values. As for example, for $\theta=0, g_{12}$ is zero. The second term (related to the process $\chi_{1} \chi_{2} \rightarrow f \bar{f}$ ) in $\sigma_{\text {eff }}$ in Eq. (30) is zero for $\theta=0$, whereas this is nonzero for nonzero $\theta$ value. $\Delta$ plays the role of more suppression of the effect of this process on $\sigma_{\text {eff }}$ for its higher values. The third term (related to the process $\left.\chi_{2} \chi_{2} \rightarrow f \bar{f}\right)$ in $\sigma_{\text {eff }}$ is more exponentially suppressed than the second term for higher $\Delta$ values. So, if $\Delta$ values are increased further than those considered in Fig. 4, there will be further lesser effect from second and third terms in $\sigma_{\text {eff }}$. For smaller $\Delta$ values much lesser than 1, there would have been more effect from the second and third terms in $\sigma_{\text {eff }}$ in Eq. (30) due to coannihilation. One may note however that active and sterile neutrino mixing constrains both $\Delta$ and $\theta$ simultaneously, as shown in Fig. 2, and for $\Delta$ less than about 1, there is no allowed $\theta$ value for coannihilation to occur. In Figs 4(a) and 4(b), we have chosen $\Delta=2, \theta=\pi / 4$ and $\Delta=1 \mathrm{~m} \theta=\pi / 4$, respectively, to see how much the allowed region in $M_{X}-m_{\psi}$ plane changes due to this variation of $\Delta$ for same $\theta$ value. In fact, one can see that the change is insignificant as both the figures are almost the same. However, it is also expected that there will be change in $\sigma_{\text {eff }}$ to some extent for variations in $m_{\psi_{1}}$ values also as follows from (30) but this is subject to details of the Boltzman equations and the corresponding freeze-out temperature $T$. In Figs 4(c) and 4(d), we have changed $\theta$ values to 0.4 and 1.2, respectively, with $\Delta$ fixed at 2. Comparing Figs. 4(b) $-4(\mathrm{~d})$, it is found that the allowed region changes significantly with such variations of $\theta$ value.

For freeze-out temperature $T \sim m_{\psi}$, one can see from Eqs. (30) and (31) and (8) that $\sigma_{\text {eff }}$ increase with increase in 


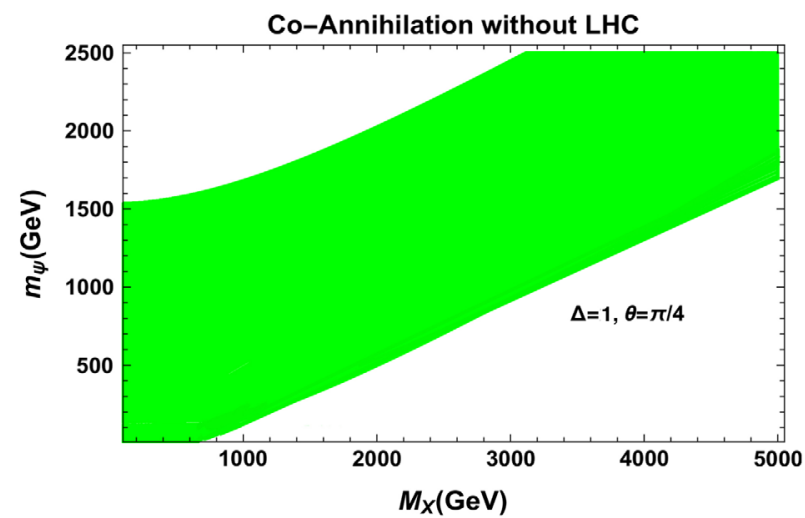

(a)

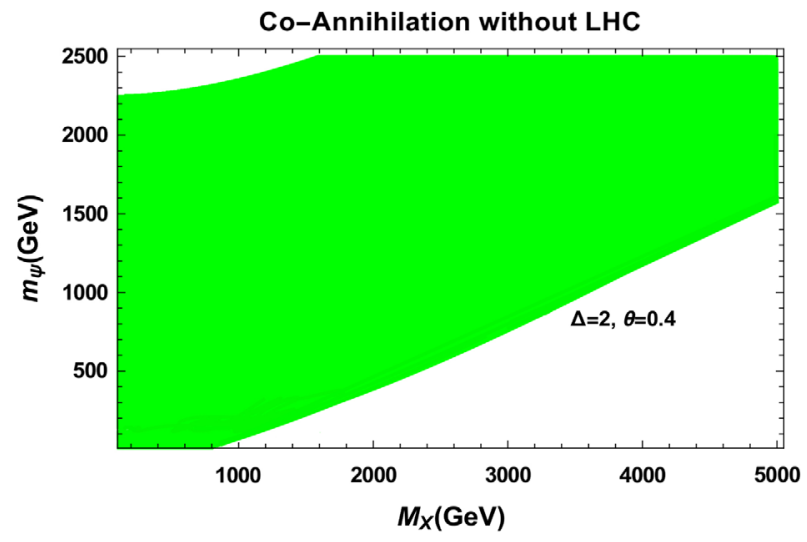

(c)

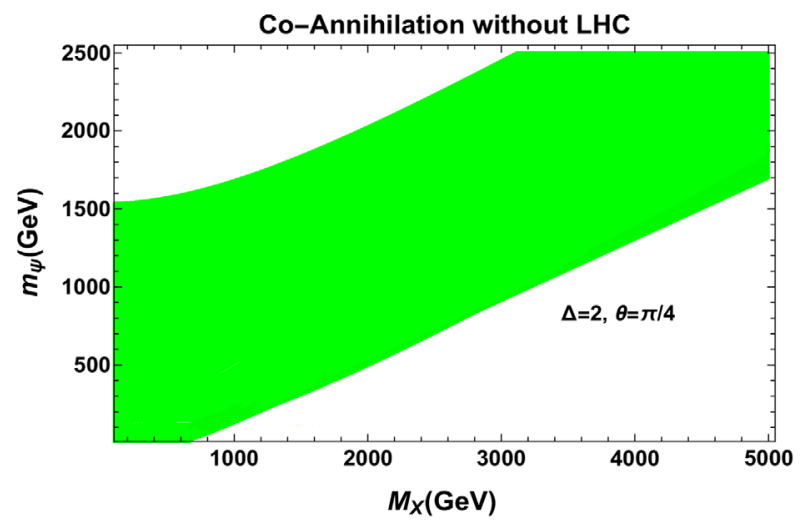

(b)

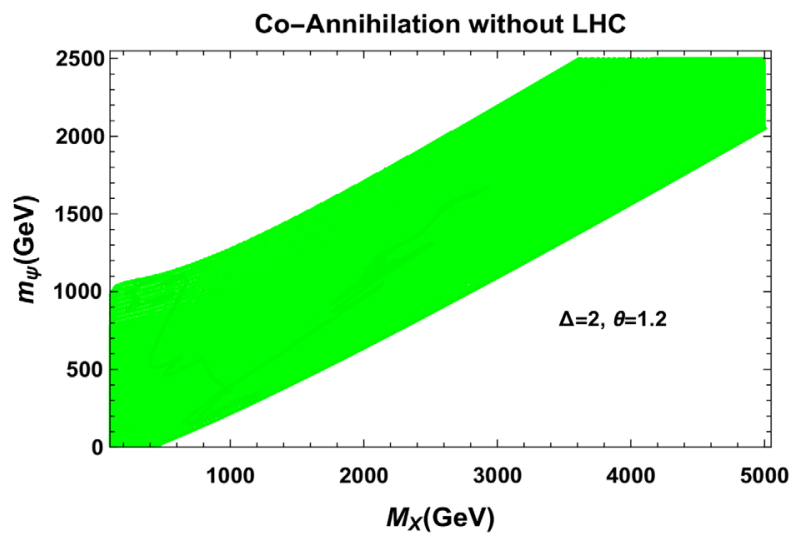

(d)

FIG. 4. Allowed green region in the $M_{X}$ and $m_{\psi}$ plane for coannihilation channels with different choices of $\Delta=2, \theta=0.4, \pi / 4,1.2$, and $\Delta=1, \theta=\pi / 4$ (in radian). The allowed region satisfies PLANCK relic density bound $\Omega h^{2} \in(0.1188,0.1212)$.

$\theta$ values and the constraint on relic density in Eq. (32) reduces the allowed parameter space of dark matter mass for any $M_{X}$ value further with increase in $\theta$ values. In Fig. 4(c), the chosen $\theta$ value is relatively smaller and the allowed region of $M_{X}$ and $m_{\psi}$ is more than that in 4(b) and $4(\mathrm{~d})$.

Figure 5 is like previous Fig. 4 but with LHC constraint on $M_{X}$ and $g_{X}$ imposed. Because of that, the allowed region for lower $M_{X}$ is very much reduced while for higher $M_{X}$ above $4000 \mathrm{GeV}$ there is more allowed region. This feature is similar to Fig. 3(b). Like Figs. 4(a) and 4(b), the allowed region is almost the same in Figs. 5(a) and 5(b). This shows that for $\Delta \geq 1$ with same value of $\theta$ there is an insignificant change in the allowed region for the same reason as discussed in the context of previous figure. With the variation of $\theta$ again, the similar feature appears like in the previous figure-namely for higher $\theta$ value there is lesser allowed region. In Fig 5(c), the chosen $\theta$ value is relatively smaller and the allowed region of $M_{X}$ and $m_{\psi}$ is more than that of in (b) and (d). Due to LHC constraint in Fig. 5, for different cases there are different lower bounds on $M_{X}$ and $m_{\psi}$ depending on different set of values of $\Delta$ and $\theta$. This feature is similar to Fig. 3(b). For higher $\theta$ value, the lower bound values for both these parameters increases to some extent with lesser allowed region. As, for example, in Fig. 5(d), the lower bound on $M_{X}$ and $m_{\psi}$ is at about 2000 and $900 \mathrm{GeV}$, respectively, which are relatively higher than those in Fig. 5(c).

Based on Figs. 3(b) and 5, we have shown the lower values of $M_{X}$ and $m_{\psi}$ in Table IV. The $g_{X}$ value as mentioned in the table has been taken from the data file corresponding to the lower values of $M_{X}$ and $m_{\psi}$ related to these figures. The vev of $\chi_{1}$ and $\chi_{2}$ fields has been evaluated using the tree-level relationship of the model as given in Eq. (5) to get an understanding of the possible scale of an extra $U(1)$ spontaneous symmetry breaking. For the coannihilation channel, the vev of $\chi_{1}$ and $\chi_{2}$ field (assuming them to be equal) is around $10 \mathrm{TeV}$, whereas for the no coannihilation channel it is around $17 \mathrm{TeV}$.

\section{HIGHER ORDER EFFECT ON $Z-X$ MIXING}

Now we address the question of $Z-X$ mixing due to higher order corrections coming from one-loop Feynman diagrams as shown in Fig. 6. The contribution from the fermions in the loop is proportional to axial-vector coupling only and as such for our choices of $n_{1}=n_{4}$ (corresponding to tree-level zero $Z-X$ mixing) the axial-vector coupling 


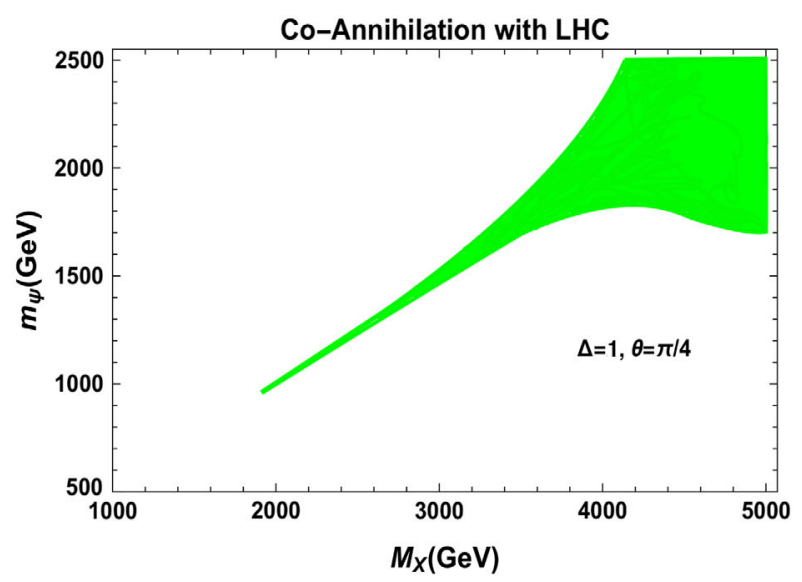

(a)

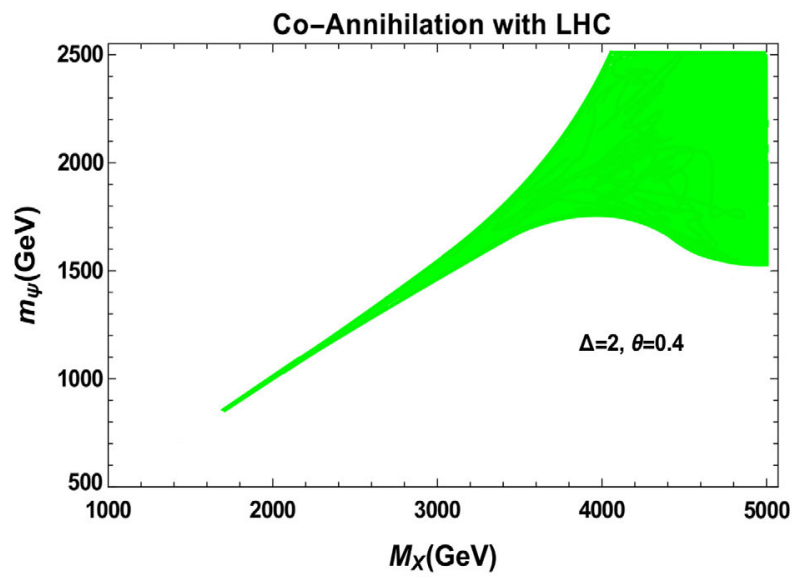

(c)

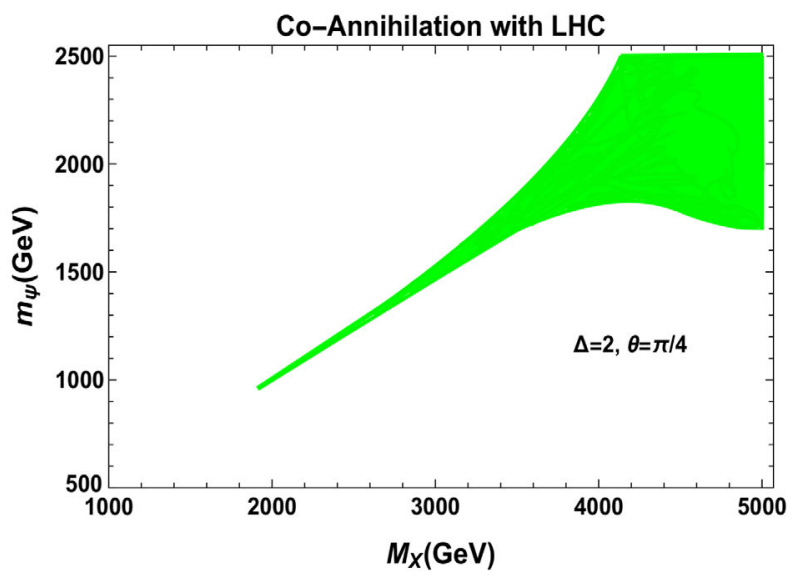

(b)

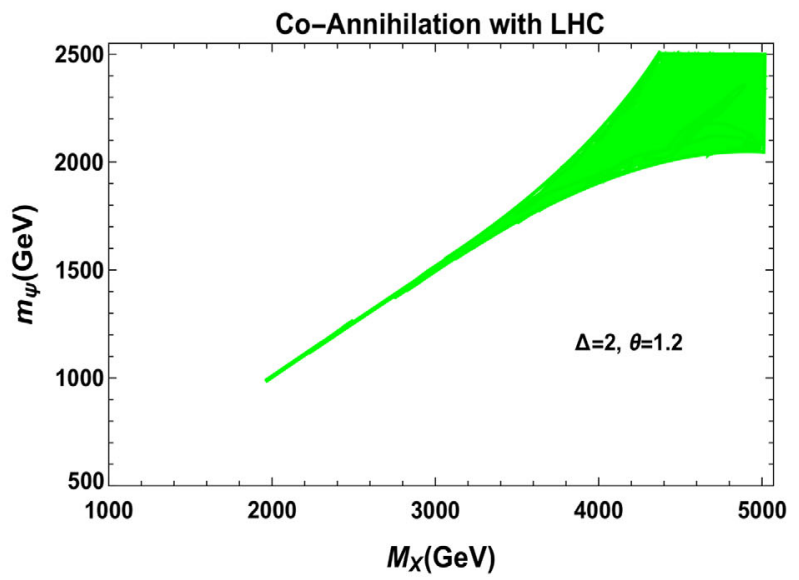

(d)

FIG. 5. Allowed green region in the $M_{X}$ and $m_{\psi}$ plane for coannihilation channels with different choices of $\Delta=2, \theta=0.4$, $\pi / 4,1.2$ and $\Delta=1, \theta=\pi / 4$ (in radian). The allowed region satisfies LHC bound and PLANCK relic density bound $\Omega h^{2} \in(0.1188,0.1212)$.

vanishes for quarks as shown in Table III giving zero contribution to $M_{Z X}^{2}$ due to quarks in the loop. The dark fermion is not possible in the loop diagram as there is no coupling of $Z$ with dark fermion due to zero $Z-X$ mixing considered at the tree level. One-loop correction to $M_{Z X}^{2}$ will have main contribution coming from $\tau$ lepton in the loop because of nonzero axial coupling for our choice of $n_{1}=n_{4}$. The general expression for one-loop correction to $M_{Z X}^{2}$ can be written as

$$
\begin{aligned}
\delta M_{Z X}^{2} \approx & -\frac{1}{4 \pi^{2}} \sum_{f} c_{A} g_{Z} g_{f_{a}} m_{f}^{2} \\
& \times\left(-\frac{1}{\epsilon}+\gamma_{e}-\log (4 \pi)+\log \frac{m_{f}^{2}}{\mu^{2}}\right) .
\end{aligned}
$$

Here $g_{Z}$ is the SM neutral coupling constant and $c_{A}$ is the coefficient depending on the particular SM fermion coupling with $Z$ boson, and $g_{f_{a}}$ is the axial-vector coupling of SM fermions with $X$ boson as given in Table III.

TABLE IV. Lower values of $M_{X}$ and $m_{\psi}$ and corresponding $g_{X}$ and vevs.

\begin{tabular}{lcccccc}
\hline \hline$\Delta$ & $\theta$ & $g_{X}$ & $M_{X}(\mathrm{GeV})$ & $m_{\psi}(\mathrm{GeV})$ & $\sqrt{u_{1}^{2}+9 u_{2}^{2}}(\mathrm{TeV})$ & $u_{1}=u_{2}(\mathrm{TeV})$ \\
\hline 2 & 0.4 & 0.021 & 1702 & 850 & 40.523 & 12.814 \\
& $\pi / 4$ & 0.035 & 1910 & 960 & 27.285 & 8.628 \\
& 1.2 & 0.025 & 1970 & 990 & 39.40 & 12.45 \\
1 & $\pi / 4$ & 0.035 & 1910 & 960 & 27.285 & 8.628 \\
No coannihilation & & 0.015 & 1645 & 825 & 54.833 & 17.33 \\
\hline \hline
\end{tabular}




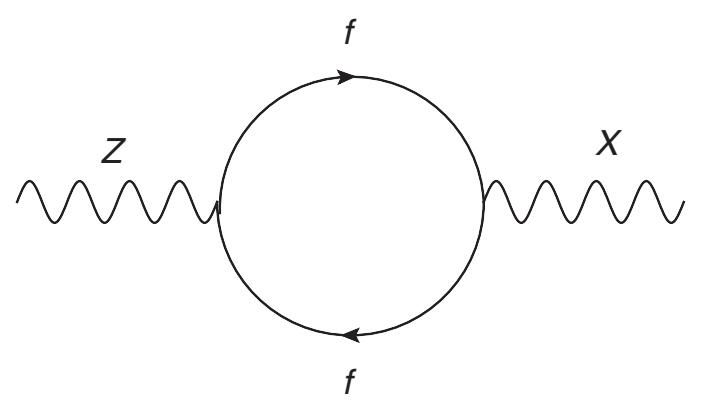

FIG. 6. One-loop Feynman diagram contributing to $Z-X$ mixing.

For completeness, we give the result for one-loop correction to $M_{X X}^{2}$. As we do not know its tree-level value from the model, this correction will not be used in our numerical computation of mixing. The main contribution to it will come from dark fermion $\psi_{1}$ and $\psi_{2}$ and also from top quark as fermion in the loop and the one-loop corrections to $M_{X X}^{2}$ due to dark matters are given by

$$
\begin{aligned}
\delta M_{X X}^{2}\left(\psi_{1}\right) \approx & -\frac{M_{X X}^{2}}{12 \pi^{2}}\left(\frac{g_{11}^{2}}{4}-\frac{3 m_{\psi_{1}}{ }^{2}}{8 M_{X X}^{2}}\right) \\
& \times\left(\frac{1}{\epsilon}-\gamma_{e}+\log (4 \pi)-\log \frac{{m_{\psi_{1}}}^{2}}{\mu^{2}}\right) .
\end{aligned}
$$

Contribution to $\delta M_{X X}^{2}$ due to $\psi_{2}$ in the fermionic loop will have similar expression like above with the replacement of $g_{11}$ by $g_{22}$ and $m_{\psi_{1}}$ by $m_{\psi_{2}}$. One-loop correction to $M_{X X}^{2}$ due to top quark and other fermions is given by

$$
\begin{aligned}
\delta M_{X X}^{2}(\text { SM fermion }) \approx & -\frac{N_{c} M_{X X}^{2}}{12 \pi^{2}} \sum_{f}\left(\frac{\left(g_{f_{v}}^{2}+g_{f_{a}}^{2}\right)}{4}-\frac{3 m_{f}^{2}}{8 M_{X X}^{2}}\right) \\
& \times\left(\frac{1}{\epsilon}-\gamma_{e}+\log (4 \pi)-\log \frac{m_{f}^{2}}{\mu^{2}}\right),
\end{aligned}
$$

in which $g_{f_{v}}$ and $g_{f_{a}}$ are given explicitly in terms of $n_{1}, n_{4}$ and $g_{X}$ in Table III and $N_{c}=3$ for quarks and $N_{C}=1$ for leptons as well as for dark fermions.

We have followed $\overline{\mathrm{MS}}$ scheme for numerical evaluation of $Z-X$ mixing. The corresponding mixing angle $\theta_{Z X}$ is given by

$$
\tan 2 \theta_{Z X}=\frac{2\left(M_{Z X}+\delta M_{Z X}\right)}{M_{X X}-M_{Z Z}} .
$$

In $M_{Z X}$, the one-loop correction is included for numerical evaluation. As an example to find a possible value of mixing, we have considered $g_{X}=0.035, \quad M_{X X}=$ $1910 \mathrm{GeV}, m_{\psi}=960 \mathrm{GeV}$ as one set of values from Table IV for lowest possible values of $M_{X}$ and $m_{\psi}$ for which mixing could be little bit larger and have used $M_{Z Z}$ as the experimentally measured value of $Z$ boson as $91.18(\mathrm{GeV})$. For such choices, we find $\theta_{Z X}=6 \times 10^{-5}$, which is quite a small number and much lesser than the possible experimental bound [6] of about $10^{-2}$, and one may consider this mixing to be almost zero with $n_{1}=n_{4}$ even after higher order correction.

\section{CONCLUSION}

Apart from Planck data constraint on relic abundance and LHC constraint on an extra $U(1)$ gauge boson mass and its gauge coupling, we have taken into account the possible constraints coming from active, sterile neutrino masses and their mixing from different oscillation experiments to find the allowed region in $M_{X}$ and $m_{\psi}$ plane. In the extra $U(1)$ gauge model considered by us, the oscillations constraints-particularly active-sterile mixing have led to the requirement of nonzero mixing $\theta$ between dark matter $\psi_{1}$ with the other heavy right-handed Majorana fermion $\psi_{2}$. This has led to the consideration of the coannihilation channel for the dark matter. Also, the oscillation data constrain the allowed region of $\theta$ and $\Delta$ as shown in Fig. 2. The allowed region in $M_{X}$ and $m_{\psi}$ plane is found to be reduced for the coannihilation channel with respect to the no coannihilation channel. Particularly, the allowed region with the coannihilation channel is sensitive to $\theta$ value as shown in Sec. V and for higher $\theta$ values with same $\Delta$ value there is lesser allowed region in the $M_{X}$ and $m_{\psi}$ plane.

The other important thing is that particularly with the LHC constraint, in general there is some kind of lower bound on both $M_{X}$ and $m_{\psi}$ as shown in Figs. 3(b) and $5 \mathrm{in} \mathrm{Sec.} \mathrm{V.} \mathrm{Using}$ the corresponding $g_{X}$ value from Table IV as followed from our numerical analysis for such lower values of $M_{X}$ and $m_{\psi}$ and using the tree-level relationship of the model as given in Eq. (5), which connects vevs of $\chi_{1}$ and $\chi_{2}$ with the mass of an extra gauge boson $M_{X}$, we get an understanding of the possible scale of the extra $U(1)$ spontaneous symmetry breaking as shown in Table IV. However, for higher values of $M_{X}$, such specific conclusion is difficult to obtain because of multiple possible values of $M_{X}, m_{\psi}$, and $g_{X}$ in the allowed region. The numerical analysis has been done considering zero $Z-X$ mixing at the tree level with $n_{1}=n_{4}$ which alters insignificantly even after including higher order corrections and satisfies various phenomenological low energy constraints. With the improvement on the constraint on the extra $U(1)$ gauge boson mass and its gauge coupling from LHC experiments, the allowed region in $M_{X}$ and $m_{\psi}$ plane could be further reduced and the lower bounds on $M_{X}$ and $m_{\psi}$ could be further higher.

\section{ACKNOWLEDGMENTS}

I. A. B would like to thank Department of Science and Technology (DST), Govt. of India for financial support through Inspire Fellowship [Department of Science and Technology, Ministry of Science and Technology, (DST/ INSPIRE Fellowship/2014/IF140038), (Republic of India/ IN)]. The authors would like to thank Sushant G. Ghosh for providing HPC facilities and Puneet Sharma for helpful computational understandings. 
[1] G. Bertone, D. Hooper, and J. Silk, Phys. Rep. 405, 279 (2005).

[2] J. C. Montero and V. Pleitez, Phys. Lett. B 675, 64 (2009); E. Ma and R. Srivastava, Phys. Lett. B 741, 217 (2015); S. Singirala, R. Mohanta, and S. Patra, Eur. Phys. J. Plus 133, 477 (2018); V. De Romeri, E. Fernandez-Martinez, J. Gehrlein, P. A. N. Machado, and V. Niro, J. High Energy Phys. 10 (2017) 169; E. Bertuzzo, P. A. N. Machado, Z. Tabrizi, and R. Zukanovich Funchal, J. High Energy Phys. 11 (2017) 004; M. D. Campos, D. Cogollo, M. Lindner, T. Melo, F. S. Queiroz, and W. Rodejohann, J. High Energy Phys. 08 (2017) 092.

[3] P. Langacker, Rev. Mod. Phys. 81, 1199 (2009).

[4] D. Suematsu and Y. Yamagishi, Int. J. Mod. Phys. A 10, 4521 (1995); M. Cvetic and S. Godfrey, [hep-ph/9504216]; A. Leike, Phys. Rep. 317 (1999); M. Cvetic and P. Langacker, arXiv:hep-ph/9707451; D. A. Demir, Phys. Rev. D 59 (1998) 015002; J. L Diaz-Cruz, J. M. Hernandez-Lopez, and J. A. Orduz-Ducuara, J. Phys. G 40, 125002 (2013).

[5] E. Ma, Phys. Rev. D 73, 077301 (2006).

[6] J. Erler, P. Langacker, S. Munir, and E. Rojas, J. High Energy Phys. 08 (2009) 017; F. del Aguila, J. de Blas, and M. Perez-Victoria, J. High Enrgy Phys. 09 (2010) 033; R. Diener, S. Godfrey, and I. Turan, Phys. Rev. D 86, 115017 (2012); J. Erler and P. Langacker, Phys. Lett. B 456, 68 (1999).

[7] F. Zwicky, Helv. Phys. Acta 6, 110 (1933); Gen. Relativ. Gravit. 41, 207 (2009).

[8] D. N. Spergel et al. (WMAP Collaboration), Astrophys. J. 148 (2003) 175; M. Tegmark et al. (SDSS Collaboration), Phys. Rev. D 69, 103501 (2004).

[9] N. Aghanim et al. (Planck Collaboration), arXiv:1807.06209.

[10] M. Aaboud et al. (ATLAS Collaboration), Phys. Rev. D 96, 052004 (2017).

[11] M. Aaboud et al. (ATLAS Collaboration), J. High Energy Phys. 10 (2017) 182.
[12] A. M. Sirunyan et al. (CMS Collaboration), Phys. Lett. B 769, 520 (2017); 772, 882(E) (2017).

[13] M. C. Gonzalez-Garcia, M. Maltoni, and T. Schwetz, Nucl. Phys. B908, 199 (2016).

[14] M. H. Ahn et al. (K2K Collaboration), Phys. Rev. D 74, 072003 (2006).

[15] P. Adamson et al. (NOvA Collaboration), Phys. Rev. D 96, 072006 (2017).

[16] A. A. Aguilar-Arevalo, Phys. Rev. Lett. 121, 221801 (2018); A. M. Sirunyan et al. (CMS Collaboration), J. High Energy Phys. 08 (2018) 130; 06 (2018) 120.

[17] R. Adhikari, D. Borah, and E. Ma, Phys. Lett. B 755, 414 (2016).

[18] N. Okada, S. Okada, and D. Raut, Phys. Rev. D 100, 035022 (2019); T. Nomura and H. Okada, Eur. Phys. J. C 78, 189 (2018); N. Okada and S. Okada, Phys. Rev. D 95, 035025 (2017); S. Singirala, R. Mohanta, S. Patra, and S. Rao, J. Cosmol. Astropart. Phys. 11 (2018) 026; Z. L. Han and W. Wang, Eur. Phys. J. C 78, 839 (2018); M. Escudero, S. J. Witte, and N. Rius, J. High Energy Phys. 08 (2018) 190.

[19] E. Ma and D.Suematsu, Mod. Phys. Lett. A 24, 583 (2009).

[20] M. Dentler, Á. Hernández-Cabezudo, J. Kopp, P. A. N. Machado, M. Maltoni, I. Martinez-Soler, and T. Schwetz, J. High Energy Phys. 08 (2018) 010.

[21] S. Vagnozzi, S. Dhawan, M. Gerbino, K. Freese, A. Goobar, and O. Mena, Phys. Rev. D 98, 083501 (2018).

[22] S. Roy Choudhury and S. Choubey, J. Cosmol. Astropart. Phys. 09 (2018) 017.

[23] M. Beltran, D. Hooper, E. W. Kolb, and Z. C. Krusberg, Phys. Rev. D 80, 043509 (2009).

[24] P. Gondolo and G. Gelmini, Nucl. Phys. B360, 145 (1991).

[25] E. W. Kolb and M. S. Turner, Front. Phys. 69, 1 (1990).

[26] K. Griest and D. Seckel, Phys. Rev. D 43, 3191 (1991).

[27] M. Cannoni, Eur. Phys. J. C 76, 137 (2016).

[28] A. Berlin, D. Hooper, and S. D. McDermott, Phys. Rev. D 89, 115022 (2014). 